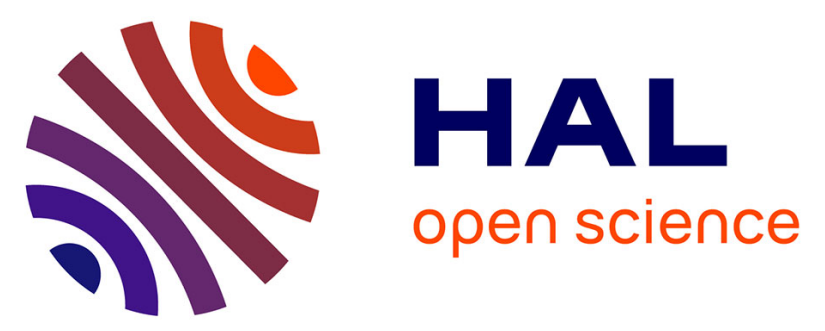

\title{
Vers une sociolinguistique française qualitative? Perspectives historiques critiques sur des processus de reconnaissance
}

Isabelle Pierozak, Didier de Robillard, Elatiana Razafimandimbimanana, Marc Debono

\section{To cite this version:}

Isabelle Pierozak, Didier de Robillard, Elatiana Razafimandimbimanana, Marc Debono. Vers une sociolinguistique française qualitative? Perspectives historiques critiques sur des processus de reconnaissance. Recherches Qualitatives, 2013, 32(1), pp.107-131. hal-01380231

\section{HAL Id: hal-01380231 \\ https://hal.science/hal-01380231}

Submitted on 12 Oct 2016

HAL is a multi-disciplinary open access archive for the deposit and dissemination of scientific research documents, whether they are published or not. The documents may come from teaching and research institutions in France or abroad, or from public or private research centers.
L'archive ouverte pluridisciplinaire HAL, est destinée au dépôt et à la diffusion de documents scientifiques de niveau recherche, publiés ou non, émanant des établissements d'enseignement et de recherche français ou étrangers, des laboratoires publics ou privés. 
PIEROZAK, I., RAZAFI, E., ROBILLARD, D. (de) et DEBONO, M., (2013), "Vers une sociolinguistique française qualitative ? Perspectives historiques critiques sur des processus historiques de reconnaissance ", Recherches qualitatives (revue en ligne), Université du Québec, Vol. 32(1) = La reconnaissance de la recherche qualitative dans les champs scientifiques, pp. 107-131. URL: http://www.recherche-qualitative.qc.ca/revue/edition_reguliere/volume32(1).html

\section{Vers une sociolinguistique française qualitative?}

\section{Perspectives historiques critiques sur des processus de reconnaissance.}

\section{Pierozak, D. de Robillard, E. Razafimandimbimanana, M. Debono ${ }^{1}$}

Isabelle Pierozak, maître de conférences, Université François-Rabelais de Tours

Maître de conférences à l'Université François Rabelais de Tours, en sociolinguistique et didactique des langues. Ses recherches sont centrées sur l'internet, envisagées sous l'angle de problématiques diversifiées, ayant comme arrière-plan constant, des réflexions épistémologiques.

isabelle.pierozak@univ-tours.fr

Didier de Robillard, professeur, Université François-Rabelais de Tours

Professeur à l'Université François-Rabelais de Tours, créoliste-francophoniste des contacts de langues, approfondissant maintenant le problème des questions épistémologiques posées par le travail de la diversité, de l'hétérogénéité, des conflits de langues et de cultures.

didier.derobillard@univ-tours.fr

Elatiana Razafimandimbimanana, maître de conférences, Université François-Rabelais de Tours Maître de conférences à l'Université François Rabelais de Tours, ses travaux de recherche visent à interroger les constructions identitaires, les dynamiques plurilingues, (pluri)migratoires et les enjeux épistémologiques en sociolinguistique.

elatiana.razafi@univ-tours.fr

Marc Debono, maître de conférences, Université François-Rabelais de Tours

Maître de conférences à l'Université François Rabelais de Tours en sociolinguistique/didactique des langues-cultures, ses recherches portent sur la didactique de l'interculturel, l'épistémologie de la diversité en didactique des langues-cultures et en sociolinguistique.

marc.debono@univ-tours.fr

\section{Résumé}

Cet article s'inscrit dans le droit fil des travaux d'analyse critique de la recherche en sociolinguistique de l'EA 4246 PREFics-DYNADIV. Il s'inspire d'une réflexion herméneutique (Gadamer notamment) pour considérer la sociolinguistique française, de manière prioritairement épistémologique. L'objectif est donc de se demander si la réflexion méthodologique n'y tient pas souvent lieu d'épistémologie. L'usage de ces deux termes clefs ( « méthodologie » et " épistémologie»), dont les diverses conceptions et mises en pratique

\footnotetext{
${ }^{1}$ L'ordre de signature des auteurs s'est construit dans le cadre d'un travail commun plus largement mené au sein de l'équipe de recherche PREFics-DYNADIV (EA 4246) et pour lequel une alternance a été envisagée à des fins d'équilibre entre les contributeurs. Dans le présent texte, après co-élaboration, la première partie a principalement été rédigée in fine par I. Pierozak et E. Razafimandimbimanana, la deuxième par M. Debono et la troisième par D. de Robillard.
} 
seront problématisées, n'est en effet pas anodin pour une recherche se revendiquant comme qualitative.

Nous adopterons, à cet égard, une perspective à la fois historique (modèles et héritages convoqués, interprétés, négligés) et critique, en s'interrogeant en particulier sur les manières qu'ont les sociolinguistes de construire du sens et les enjeux qui y sont attachés, en particulier en termes de reconnaissance.

Mots clés : Sociolinguistique - France - Herméneutique - Epistémologie - Qualitatif 


\section{Vers une sociolinguistique française qualitative?}

\section{Perspectives historiques critiques sur des processus de reconnaissance.}

\section{Introduction}

C'est une véritable gageure que de prétendre traiter, en si peu de place, ce que laisse entrevoir l'intitulé de cet article. Nous avons dû faire des choix, confortés par la lecture de l'appel à contributions, qui incite à se concentrer sur un champ, et/ou les usages admis dans la littérature scientifique. Il en va donc ainsi du champ de la « sociolinguistique française »(SF), appellation que nous trouvons, ne serait-ce que pour la dernière décennie, sous la plume notamment de Calvet (2000), Boutet (2000), Bulot (éd, 2001), Gadet (2005), Boyer (éd. 2010), malgré des difficultés définitoires inhérentes, tant les critères sont à géométrie variable. Ce champ ne préexiste pas d'emblée, mais est (re)construit a posteriori, selon une certaine perspective historique, à questionner. De même et pour terminer la glose de l'intitulé, nous utiliserons le terme de « légitimation » mais aussi celui de « reconnaissance », pour élargir les classiques références sociologiques en la matière ( $c f$. notamment Bourdieu et Passeron, 1970) aux références philosophiques ( $c f$. Garrau et Le Goff, 2009), non exposables ici.

$\mathrm{Au}$ fil du texte, nous avons choisi d'argumenter les principaux éléments suivants. Tout se passe comme si la SF, dans une forme d'ambition d'autonomisation vis-à-vis de la linguistique, se rangeait sous l'étiquette du « qualitatif», mais sans l'expliciter, et donc sans questionner ce qu'elle recouvre, et sans s'interroger du même coup, sur les conséquences induites. Le propos n'étant pas de proposer une histoire, mais plus modestement, au regard du volume imparti, de questionner ce manque, on commencera par problématiser l'absence de récits historicisants de la SF pour ensuite poser la question de ses processus de reconnaissance, et enfin exposer des éléments de réflexions puisés dans le vaste corpus de littérature phénoménologico-herméneutique $(\mathrm{PH})$. Le lecteur mesure à présent la gageure de ce texte, pour le moins nécessairement condensé au regard du propos couvert.

\section{Quelle(s) histoire(s) de la SF ?}

\subsection{Une histoire au second plan}

En comparaison de ce que l'on peut constater dans d'autres champs, la littérature scientifique de la SF semble réserver bien peu de place à son histoire. Le projet d'un Sylvain Auroux (1994), pour les sciences du langage, ne semble pas ainsi avoir eu d'équivalent pour la 
sociolinguistique. Ce déficit ne signifie pas pour autant une absence de ce type de réflexions. Mais ce qui ressemble à un rituel ( $c f$. le genre des thèses) semble servir à articuler la position délicate des sociolinguistes, à la fois dans et en-dehors des sciences du langage. Savatovsky (2011) souligne encore aujourd'hui la difficulté pour les sociolinguistes à se faire reconnaître et recruter dans les universités françaises. On comprend mieux dès lors le manque de problématisation approfondie de ce rituel, et cela était/est assez symptomatique de l'inconfort d'être sociolinguiste en France.

Si l'on en vient à présent au profil récurrent de cette Histoire, on peut se reporter à Boyer (2001) particulièrement intéressant ici puisque destiné aux néophytes. Cet ouvrage témoigne exemplairement, dans le $1^{\mathrm{er}}$ chapitre subdivisé en deux parties, de comment l'histoire de la SF se donne à voir conventionnellement, autour de quatre grands points incontournables ${ }^{\mathrm{ii}}$. Il s'agit d'une Histoire généralement présentée comme :

1. récente : les quelques décennies de rétrospective se comptent sur les doigts de la main,

2. «(tout) contre » Saussure : la figure d'un Meillet est ainsi parfois convoquée,

3. grandement tributaire de Labov, en tant que permettant d'asseoir une « sociolinguistique [dite] moderne », par exemple chez L.-J. Calvet (1999),

4. et particulièrement hétérogène, ce qui régulièrement donne lieu à deux types de problématisations interdépendantes : les affinités disciplinaires et la dénomination de la «sociolinguistique », mise en concurrence avec d'autres dénominations, comme «sociologie du langage», ou pluralisée en courants, avec par exemple la « sociolinguistique interactionnelle », etc.

Ce profil historique récurrent peut être lu comme une configuration d'éléments repères répondant à des besoins, intérêts, sur lesquels il nous faut nous interroger. En l'occurrence, pourquoi, dans la littérature sur la SF, est-il rare de proposer une histoire qualitativement située?

Nous y reviendrons mais au préalable, une dernière mise en cohérence s'impose : si la SF fait si peu de cas de ses histoires possibles, cela semble bien avoir pour corollaire de constantes interrogations quant aux domaines et objets de la sociolinguistique. On peut ainsi avancer que les questionnements historiques de la SF prennent plutôt la forme, non pas d'une histoire plurielle, mais d'une juxtaposition de domaines et objets dans le temps (Laks, 1984 ; Boyer, 1996 ; Boutet, 2000) $)^{\mathrm{iii}}$. Or cette façon de mettre en récit le champ pose un débat au plan 
épistémologique, car l'objet est effectivement un enjeu, mais essentiellement sous un angle réaliste. Qui plus est, privilégier «l'objet» des travaux de sociolinguistique, comme on continue de le faire ( $c f$. sur différents «niveaux »: les parlers jeunes, la sociolinguistique urbaine, l'intervention sociale, la violence verbale, le langage et le travail, etc.) permet de comprendre pourquoi, même si l'on dit s'écarter d'une approche par trop positiviste de la sociolinguistique, l'on peut finir par croire, en ce qui est présenté comme son corollaire métonymique, à savoir « le terrain » sur lequel nous reviendrons.

\subsection{Questions en suspens et pistes de réflexions}

Ce qui nous apparait comme un problème à s'historiciser appelle deux ensembles de questions, organisables autour des problématiques du «pourquoi » et du «comment» (cf. aussi Robillard (2010), en lien avec la dernière partie de ce texte). Pourquoi les sociolinguistes semblent-ils accorder si peu de place à une histoire plurielle de la SF ? Et comment un champ peut-il fonctionner ainsi?

Au plan du pourquoi, plusieurs pistes sont formulables. Parmi les plus immédiates, on peut

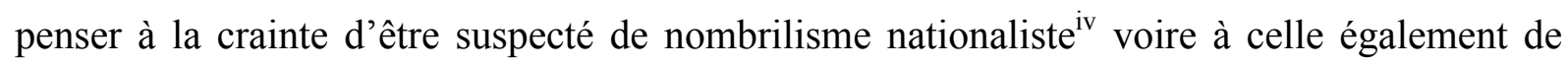
rompre un silence tacite face à des questions dont l'analyse pourrait avoir des effets délétères. Ou s'agirait-il plutôt d'un obstacle épistémologique à parler de ce qui est trop proche, car de l'ordre de sa propre histoire ? Ou serait-ce la crainte de produire une image dévalorisée de la SF, symptomatiquement présentée comme «éclatée » (Boyer, 2001) vs «foisonnante» (Calvet, 2011), pour renvoyer aux seules références de base préalablement citées ? On pourrait aussi évoquer la crainte d'une priorité historique pensée comme très secondaire face aux récurrents problèmes de définition des frontières disciplinaires et des objets d'études. Sans doute, toutes ces raisons, seraient à réfléchir plus avant. En somme, c'est aussi l'image de ce que devrait être un champ de recherche qui est en cause. Et si dans les représentations, celui-ci doit nécessairement être unifié, cela pourrait délégitimer d'autres histoires de la sociolinguistique qui, sans nier les différences, essaieraient de les articuler.

Il est une autre piste envisageable au plan de la reconnaissance, se déclinant en deux temps. Dans un premier temps, la SF, pour se faire reconnaître, se serait abritée derrière une autre histoire, anglo-américaine en citant par exemple les noms de Bernstein est britannique, Fishman, Gumperz, Hymes, Labov. Faire appel à l'autorité de ces auteurs, présentés comme des incontournables, c'est s'attribuer l'histoire des autres, qui présente en l'occurrence 
l'intérêt d'être « délocalisée », ce qui légitimerait donc de ne pas se poser prioritairement la question de son histoire propre, et par là même, d'éviter les concurrences locales ${ }^{\mathrm{v}}$. En ce sens, il serait plus supportable de se confronter à des altérités lointaines plutôt que proches. Dans un second temps, ce positionnement de la $\mathrm{SF}$, en viendrait à faire de l'ombre aux traditions antérieures, anté-saussuriennes, et qui ressurgiraient donc dans des espaces «attendus» (certains manuels ou thèses) surtout lorsque la dimension historique est clairement assumée (Baggioni, 1986).

Une dernière piste nous ramène à la problématique du qualitatif. Le peu d'histoire donné à voir en SF semble comme vidé de tout enjeu sensible pour les individus sociolinguistes. Quels enjeux pourrait-il y avoir à ne pas travailler cette histoire à partir des expériences des sociolinguistes ( $c f$. supra les concurrences locales, et voir aussi infra) ? Ce choix semble avoir comme conséquence de permettre de penser méthodologiquement le qualitatif et le quantitatif de manière complémentaire, en gommant les enjeux qu'une approche qualitative oblige à formuler et qui mettrait le chercheur au cœur des choix opérés pour sa recherche. Historiquement d'ailleurs, la reconnaissance du qualitatif en SF s'est faite de manière indirecte, puisque la SF est de nouveau allée chercher de la méthodologie qualitative (plutôt que de l'épistémologie) ailleurs, à savoir en sociologie (notamment ethnométhodologie), anthropologie, etc., pour s'affranchir de la tutelle quantitative de la linguistique ( $c f$. la partie suivante).

$\mathrm{Au}$ plan des conséquences, se pose aussi la problématique du «comment » la SF fonctionne avec si peu d'historicisation critique; plus précisément, comment peut-elle se concevoir, s'assumer et se projeter? Pour ce qui est de sa conception, les récurrents questionnements qui se posent autour des domaines, «terrains » et objets peuvent être vus comme symptomatiques de l'absence de ce travail historique, comme nous l'avons vu précédemment. En effet, dans une optique herméneutique ( $c f$. la dernière partie du texte), on voit mal comment ce questionnement sur l'histoire pourrait être écarté de ces problèmes présentés à juste titre comme définitoires de la SF, qui s’évertue ainsi à chosifier son « être ».

Pour autant, la $\mathrm{SF}$ « fonctionne » mais sa projection réflexive n'est pas à vrai dire pensée, et consiste surtout dans ce qui nous semble être un interventionnisme réflexe. L'histoire du Réseau «Français» de Sociolinguistique, devenu «Francophone» en 2008 est particulièrement exemplaire de temps forts interventionnistes, qui ont contribué, en une 
dizaine d'années, à souder une communauté de chercheurs grandissante, et finissant par déborder, dans ses dynamiques, les seules frontières françaises ${ }^{\mathrm{vi}}$. Mais ce type d'actions, au coup par coup et réaffirmant classiquement une identité contre autre chose, et faisant de son unité une condition indispensable, semble empêcher une pensée plus subtile, qui articulerait des courants différents, avec leurs passés, pour des actions diversifiées. Nous lisons donc ici ce défaut de vision(s) politique(s) (nuancées, débattues), au profit d'actions éparses recherchant l'unanimité, comme venant en quelque sorte compenser un manque à penser épistémologique ${ }^{\mathrm{vii}}$, régulièrement masqué par les questions méthodologiques où qualitatif et quantitatif finissent par se compléter et s'équivaloir ( $c f$. la partie suivante).

Pour clore là ce paragraphe, il faut souligner que ne pas se confronter à son histoire, pour quelque raison que ce soit (et plusieurs ont été évoquées) et quelque conséquence que cela ait, ne peut que rendre encore plus difficile les processus de reconnaissance - la reconnaissance d'un champ et a fortiori la reconnaissance des recherches qualitatives dans leur diversité en SF. On verra qu'en ce qui nous concerne, l'une ne va pas sans l'autre.

\subsection{Une histoire nécessairement interprétative : quels enjeux ?}

L'histoire de la SF ne sera donc pas envisagée comme s'il était possible de retracer la totalité des événements constitutifs pour en faire un récit unique, factuel, linéaire. Nous partirons plutôt de l'idée inverse, c'est-à-dire que l'histoire de la SF est impossible si ce n'est à partir d'une approche interprétative et donc, située, sélective, intersubjective mais néanmoins articulée pour affirmer qu'une telle historicisation contribuerait aux processus de reconnaissance. Argumenter cela revient à dire que l'Histoire mise en récit par les sociolinguistes relève déjà d'interprétations. La multiplicité des origines citées par les sociolinguistes, tant sur le plan des fondateurs et des dates repères que sur celui des disciplines tutélaires, sur lesquelles nous reviendrons, témoigne en effet des histoires possibles, c'est-à-dire d'une histoire plurielle, ce qui ne signifie pas « désarticulée ».

Une première interprétation des origines de la SF consiste à les rattacher à la France tandis qu'une deuxième en fait une importation américaine. Dans les deux cas, l'absence de consensus permet d'y voir des interprétations différenciées de ce qui est signifiant (ou pas) pour chaque sociolinguiste sachant que son positionnement est en partie façonné par son parcours, ses rencontres, sa réflexivité et ses projets (sociaux, intellectuels, professionnels, etc.). Lorsque situées en France, les origines de la SF coïncideraient tantôt avec l'enquête dialectologique de l'Abbé Grégoire en 1790 (de Certeau et al., 1975, p. 13 ; Boyer, 1990), 
tantôt avec les travaux réalisés un siècle plus tard par Meillet (Marcellesi, 1980) aux « intuitions de type sociolinguistique » (Trimaille, 2003, p. 13). Selon d'autres perspectives, l'émergence de la sociolinguistique en France correspondrait aux années cinquante et soixante-dix et là encore, il y a une diversité d'acteurs clés dans les références mobilisées. On pourra voir Cohen, Calvet, ou le binôme Marcellesi et Gardin (Boutet et Tabouret-Keller, 2009). Il en va de même quant aux événements décrits comme symboliques de cette émergence, les repères incluent la publication de l'ouvrage Introduction à la sociolinguistique de Marcellesi et Gardin en 1974 (Marcellesi et al., 2003) ; la sortie du numéro 46 de la revue Langage en 1977 (Gadet, 2005) ou la tenue du colloque «Pratiques linguistiques, pratiques sociales » de Rouen en 1979 (Boutet et Tabouret-Keller, 2009 ; Marcellesi, 1980).

Lorsque les origines de la SF sont «délocalisées », Labov détient une place quasi incontestée tant les qualificatifs qui lui sont attribués sont univoques. On peut en effet lire qu'il est « exemplaire », « père », « pionnier», « fondateur» (Boyer et Prieur, 1996: 42 ; Oliveau-Statius, 2006 ; Trimaille, 2003, p. 17), voire «prophète » (Gregersen, 2010). Ce qui peut suggérer la croyance en une origine unique et universelle de la sociolinguistique, or nous arguons que la SF s'est construite à travers des processus interprétatifs selon les projets de chaque chercheur et de chaque courant. Différentes filiations apparaissent ainsi en fonction des contextes, par exemple Bernstein, Bright, Labov sont convoqués par Calvet (2002, p. 1420), tandis que Hymes, Labov, Fishman le sont par Klinkenberg (1997, p. 82) ou encore Saussure, Meillet, Martinet, Weinreich, Labov dans Koerner (1991).

Ecrire l'Histoire reviendrait à (im)poser une seule «expérienciation » ${ }^{\text {viii }}$ et par conséquent, une seule forme de légitimité de la SF. L'impossibilité de cette histoire-là est évidente si l'on considère, outre la polarisation des origines mises en récit, la pluralité revendiquée par certains sociolinguistes face à leurs parcours (Blanchet, 2000 ; Blanchet et. al., 2007 ; Marcellesi et. al., 2003 ; Razafimandimbimanana, 2005). Toutefois, compte tenu de la place restreinte que les chercheurs accordent aux réflexions épistémologiques qui explicitent, historicisent leurs positionnements en SF, d'importants enjeux y sont sans doute associés, comme nous l'avons déjà mentionné. En ce sens, l'absence notoire de toute historicisation (du chercheur et/ou du champ) dans Boyer (1996) et Moreau (1997) laisse supposer, pour le premier que traiter du «territoire» (dont on notera le singulier) et des « objets » reviendrait à définir le champ comme ayant une unité fondamentale, et une pluralité de retombées. Cela implique-t-il qu'une histoire est réductible à un territoire et à des objets prédéfinis? L'avant-propos du second ouvrage situe le champ comme étant «arrivée à sa 
maturité » (1997, p. 5), cela exempte-il les sociolinguistes d'un travail problématisé sur leurs histoires (individuelles et collectives) et/ou d'une réflexion intégrant celles-ci ? Il semble y avoir davantage de risques que de bénéfices à reconstituer une histoire de la SF. L'un des risques encourus serait effectivement d'être soupçonné de parti-pris caractérisé par les tenants des divers courants, etc. Pour nous, écrire l'histoire de la SF revient nécessairement à en écrire plusieurs, chacune mettant en débat les positionnements, «manières d'être » ( $c f$. la dernière partie) et projets (des chercheurs et de leurs réseaux) qui ne peuvent faire autrement que de participer à l'historicisation de leur discipline. Nous avons vu que l'absence d'historicisations explicitées ne signifie pas l'absence d'histoires surtout si on accepte de se représenter l'histoire comme étant simultanément une mise en cohérence entre un passé et un devenir, la reconstruction d'une pensée collective mais aussi comme un discours de légitimation : «L'histoire est le récit cohérent de ce que l'on est en mesure de reconstituer à la suite d'un travail d'enquête systématique et de déduction vraisemblable, ou ce que l'on s'autorise à dire parce que l'on détient un pouvoir que l'on veut continuer d'exercer » (Jewsiewicki et Létourneau, 1996, p. 15). Pour des sociolinguistes qui se définissent comme qualitatifs, se pose immanquablement alors la question quant au sens donné au « qualitatif ». Ce qui nous engage à chercher à comprendre les références et arrière-plans du paradigme qualitatif.

\section{Quelle(s) conception(s) du qualitativisme en SF ?}

\subsection{Problématisations implicites et néanmoins agissantes}

Ce qui sera écrit plus bas doit être s'interpréter comme un état, non pas du champ «SF» artificiellement homogénéisé, de la plupart des chercheurs se réclamant de ce champ. En effet, s'il ne fait pas de doute que certaines individualités (Gadet, Boutet, Blanchet parmi d'autres) se préoccupent d'épistémologie, il n'en demeure pas moins que cette activité est marginalisée au sein du champ ( $c f$. les éléments considérés comme centraux lors de la formation des doctorants, des thèmes de nombreux colloques, etc.). Ce déficit épistémologique s'explique d'au moins deux façons. La diffusion du pragmatisme et des retombées de la philosophie analytique en France, courants fondés sur des bases déniant toute pertinence aux grandes questions traditionnelles de la philosophie, a poussé à une forme de croyance dans un empirisme peu argumenté épistémologiquement alors que, au sein de ces courants, ces positions empiristes sont fondées sur des argumentaires sophistiqués (ceux du second Wittgenstein, ceux de Peirce, de Mead, ou ceux de Dewey par exemple). Lorsqu'un chercheur 
de la stature de P. Bourdieu argumente contre la skholè (Bourdieu, 1997), il recourt, paradoxalement, lui-même à l'épistémologie pour expliquer pourquoi on pourrait s'en passer, et mobilise la tradition philosophique pour le faire. Cela n'a pas empêché des interprétations réductrices de cet argumentaire (lorsqu'il est connu), qui conduisent une majorité de sociolinguistes à marginaliser, avec une bonne conscience appuyée sur la notoriété d'un P. Bourdieu simplifié, les débats épistémologiques, sans que les éléments de ce débat complexe, et toujours ouvert, soient réellement connus.

La problématisation du qualitatif est ainsi souvent considérée comme une question simple. «Qualitatif » serait implicitement le contraire de « quantitatif», l'absence de chiffres vaudrait qualitativité.

Ce positionnement «hyper-empiriste » (Boltanski, 2011) a des fonctions pragmatiques : le déficit de réflexion sur ce plan permet sans trop de difficultés de justifier des positionnements œcuméniques qui permettent d'éviter des controverses avec les linguistes plus classiques, avec lesquels, pour des raisons institutionnelles qui ne seront pas évoquées ici, les sociolinguistes sont souvent appelés à collaborer.

De manière assez contradictoire, cela conduit donc des sociolinguistes œcuméniques à faire comme si les approches des questions linguistiques pouvaient être politiquement neutres, ce que dément l'histoire de la (socio)linguistique : le processus de "grammatisation » résulte en un immense «linguicide» (Auroux, 1994, p. 116). La non problématisation approfondie du qualitatif résulte également en un réalisme épistémologique implicite (la réalité existerait indépendamment des chercheurs, ce qui permettrait de «croiser les regards sur elle» de manière complémentaire, et jamais contradictoire). Cela est souvent incohérent avec les usages qui sont faits de notions telles que « constructivisme», ou « représentations ». Cette incohérence constitue une autre source de délégitimation pour la SF pour qui se plonge avec un peu de rigueur dans les propos des sociolinguistes.

Le qualitatif est parfois problématisé, différentiellement. Pour s'opposer aux linguistes, les sociolinguistes considèrent souvent que leur méthodologie, leurs outils (par opposition à la méthode (Morin, 1986, p. 27)) définiraient le caractère qualitatif de leur travail. Ainsi, le travail par entretiens ou étude d' « interactions » serait par nature qualitatif. Il est clair que ces observables peuvent être utilisés de manière quantitative, de même qu'un tableau de chiffres peut se voir interpréter qualitativement (ex. Pelletier et Demers, 1994).

Un autre angle d'attaque consiste à considérer que le qualitatif se définirait par un faisceau de traits. Le plus saillant serait celui de l'objectif d'intervention pratique, que défendent nombre 
de sociolinguistes (ex. Blanchet, 2012) : problèmes scolaires, politique linguistique, langues du travail, violence verbale, etc. ( $c f$. la première partie du texte). Cet objectif imposerait, avec des attitudes psycho-affectives telles l'empathie, la nécessité de comprendre les motivations des acteurs, la recherche du sens « émique », une posture humaniste (terme rarement défini), ou encore des options politiques, attitudes de gauche et ou marxistes, défense des plus faibles, notamment face aux situations néo-indépendantes. Le qualitatif se problématiserait donc par sa volonté d'efficacité pratique dans les champs social et politique. Ce type de critère sousjacent ne résiste pas beaucoup à l'analyse : les objectifs d'intervention peuvent difficilement rendre obligatoires des choix méthodologiques, d'autant qu'on pourrait argumenter que même les approches les plus quantitatives se construisent sur des catégories qui, elles, sont nécessairement qualitatives à la base.

On s'aperçoit donc que "qualitatif», au sein de la SF, est une notion peu élaborée conceptuellement, ce défaut d'élaboration ayant des causes et fonctions diverses comme on l'a vu. Il est temps de se demander, puisque la problématisation de la SF qualitative n'est pas faite explicitement intellectuellement, comment elle se vit dans les faits. Boutet (2006, p. 1104) souligne les origines marxistes de la SF, problématisations qui ont été minées à la fois par les critiques de Labov et Bourdieu. La situation qui en est issue est confuse : Bourdieu continue, par sa sociologie, à influencer la SF, avec cependant des greffes peu réfléchies conceptuellement et parfois peu compatibles entre elles, celles de différents courants inspirés du pragmatisme et/ou de la pragmatique, sur un fond ancien de labovisme positiviste. On peut argumenter que ces courants ne sont pas quantitatifs (l'inspiration labovienne reste plus quantitative), mais il faudrait justifier leur caractère qualitatif avec des arguments très différents. Mis à part les différents courants fortement attachés aux descriptions de formes linguistiques (l'approche de Gadet en est un bon exemple), la grande majorité des sociolinguistes justifie son activité, plus que par des considérations épistémologiques, par des objectifs citoyens, politiques : égalité, justice sociale etc., nourris par la forte passion française pour l'égalité, des vestiges de morale religieuse, une certaine mauvaise conscience post-coloniale qui poussent à agir « sur le terrain » et/ou sous couvert d'un humanisme bien-pensant, pour les dominés. Dans la mesure où la SF considère, à quelques exceptions près, que son épistémologie se fait dans ses disciplines tutélaires (sociologie, linguistique, anthropologie) ${ }^{\mathrm{ix}}$, les débats épistémologiques sont $\operatorname{rares}^{\mathrm{x}}$, et ce déficit laisse le champ libre à ces diverses problématisations, incohérentes entre elles.

Cela est dommageable à la clarté des débats, et à la reconnaissance de la discipline : en effet, celle-ci, avant de pouvoir rechercher la reconnaissance par les acteurs de son champ, doit au 
moins satisfaire aux critères minimaux reconnus dans la sphère de la recherche, à savoir un discours critique, historicisé, informé et cohérent sur sa propre prétention à l'existence. Quelques efforts en ce sens sont néanmoins repérables.

\subsection{Les trois piliers du qualitativisme sociolinguistique}

Henwood et Pidgeon (1994, cité dans Drapeau, 2004) ont proposé comme triptyque constitutif de la recherche qualitative l'empirisme, le contextualisme et le constructivisme ${ }^{\mathrm{xi}}$. Le qualitativisme sociolinguistique étant largement «emprunté » à d'autres disciplines, rien d'étonnant à ce que l'on y retrouve ces fondations transversales aux SHS. Nous montrerons l'importance de ces trois « piliers » en SF, tout en en pointant certaines limites, annonciatrices de notre conception du qualitatif «phénoménologique-herméneutique » $(\mathrm{PH})$ exposée plus bas.

\subsubsection{L'empirisme : terrain et corpus}

Pour les sociolinguistes, l'ancrage empirique de toute recherche est un précepte fondateur : ils se définissent avant tout par rapport à leur terrain, leurs données, et on oppose traditionnellement l'empirico-inductif qualitatif à l'hypothético-déductif quantitatif/positiviste (cf. Blanchet (2000) qui l'emprunte à Martinet).

Parce que l'empirisme s'oppose à l'hypothético-déductif, dont la figure serait, pour les sociolinguistes, Chomsky et sa «linguistique cartésienne », on a cru qu'en se réclamant de l'empirisme et de la méthode inductive, on échappait aux pièges du chomskysme, qui, selon Calvet (1999), contribue fortement par sa montée en puissance, à cristalliser ce qu'on allait appeler la sociolinguistique ${ }^{\mathrm{xii}}$.

En sciences du langage, l'empirisme se concentre autour de deux notions : historiquement, « corpus, » puis, avec le contextualisme, « terrain », sorte d'aura diffuse (Robillard et al., à paraître ; Goï et Pierozak, 2010). À travers ces notions clefs, l'empirico-inductif devient un élément marquant. Ce "pilier» s'inscrit pourtant dans une perspective sémiotique qui ne varie pas par rapport à la linguistique, perspective séparant les signes (les corpus, les «données brutes » issues du terrain) de l'acte d'interprétation. Or cette approche s'oppose assez nettement au qualitativisme PH développée plus bas : «[...] l'orientation empiriste est nécessairement hostile à une attitude de recherche basée sur l'interprétation » et relève d'un « idéal vérificationniste » jugé délétère en SHS par Taylor (1997). En ce qu'il suppose et survalorise des «données brutes » sources de vérification et séparables des chercheurs- 
interprétants, l'empirico-inductif de la SF entre donc en tension avec une démarche interprétative (induire pour « vérifier » n'est pas interpréter). Nous verrons que cet empirisme revendiqué entre également en tension avec l'hypothèse constructiviste en sociolinguistique (induire ou construire le sens ?).

\subsubsection{Le contextualisme ou comment sécuriser le sens}

La linguistique ayant pour principe de base de décrire tout ce qui est indépendant des contextes, dans un jeu d'opposition « constituant » la discipline tutélaire, la sociolinguistique se veut qualitative en contextualisant. L'idée de cette approche est que, contextualisé, le sens des données sera plus précis, clair, riche, évident. Le contexte est donc bien souvent compris de manière réductrice et techniciste comme un moyen de désambiguïser le sens en « entourant» les signes d'éléments réputés objectivables : cadre matériel de l'interlocution, les rapports de pouvoir, etc. (mais ces éléments ne sont-ils pas eux-mêmes sources de potentielles interprétations variées ?). Le contextualisme sociolinguistique participe donc de l'objectivisme : le contexte reste un objet dont le rôle est de présenter un autre objet (les données constituées en corpus).

Or, ne peut-on avancer que, si tout le monde s'accorde sur la possibilité/nécessité de discuter de l'attribution de significations au corpus, le contexte joue souvent le rôle d'argument d'autorité ${ }^{x i i}$ ? Une conception objectiviste de la contextualisation permet au chercheur de ne pas se référer explicitement à son expérience ${ }^{\text {xiv }}$, ce qui pose la question de la compatibilité du contextualisme avec le constructivisme, autre pilier du qualitativisme SF.

\subsection{3. (Co-)constructivisme : un statut ambigu, entre méfiance et rationalisation}

Le constructivisme, tel que pensé par les théoriciens majeurs de ce courant que sont Piaget et Watzalwick (Palo Alto), est une théorie qui repose sur l'idée que nos connaissances ne sont que le produit de l'entendement humain et non le reflet exact de la réalité. Il constitue donc une réaction radicale à l'illusion réaliste du positivisme scientiste (von Glasersfeld, 1994, p. 22).

Pour les constructivistes, « connaître » c'est « se représenter » le monde non pas en cherchant une adéquation à la réalité objective mais pour agir sur lui : la connaissance constructiviste est « une construction subjective à valeur pragmatique » (Blanchet, 2000, p. 68). 
Le constructivisme est une option explicitée de la SF, classiquement envisagée comme une étude des «pratiques» inséparable de celle des «représentations ${ }^{\mathrm{xv}}$, l'idée étant que les pratiques linguistiques n'existent pas «en elles-mêmes », mais qu'elles relèvent d'une construction que les psychosociologues (à qui la notion est empruntée par les sociolinguistes) nomment « représentations » :

«Un objet n'existe pas en lui-même, il existe pour un individu ou pour un groupe et par rapport à eux. C'est donc la relation sujet-objet qui détermine l'objet lui-même. Une représentation est toujours une représentation de quelque chose pour quelqu'un » (Abric, 1994, p. 12).

Si Saussure énonçait déjà «c'est le point de vue qui crée l'objet» (2010, p. 23), la linguistique positive a cherché à développer des méthodes objectivantes, à dominante quantitative-expérimentale. À travers la valorisation des représentations linguistiques (qui ne fait pas l'unanimité chez les sociolinguistes), la SF cherche à l'inverse à réhabiliter l'étude des dimensions intersubjectives à l'œuvre dans toute interaction verbale. Reste la question méthodologique de l'inclusion ou non du chercheur lui-même dans ce paradigme constructiviste : si Calvet inclut bien le chercheur dans cette étude des représentations, et si Blanchet (2012, p. 104) évoque une "éthique de l'implication subjective » (éthique qu'il oppose à l'ambition descriptiviste de la linguistique structurale), mentionnons que le degré d'intégration d'un constructivisme qualitatif est variable au sein de la SF et qu'une méfiance tenace envers la subjectivité du chercheur tempère sensiblement cette orientation. Selon Boutet, par exemple, pour « construire le sens » (titre de son ouvrage de 1994) de l'interaction verbale qu'il étudie, le chercheur doit se fonder «non sur sa réaction subjective, mais sur les régularités formelles et observables des données à analyser »(1994, p. 28). L'empiricoinductif « objectivant» vient ici contrebalancer un constructivisme « subjectivant» : le sens vient des données (contextualisées), mais non pas du chercheur ${ }^{\mathrm{xvi}}$.

Plus fondamentalement, la SF n'échappe pas au débat sur le relativisme de la connaissance et au discrédit jeté sur le constructivisme radical (Boghossian, 2006 ; Sokal et Bricmont, 1997) : d'où la tentation chez certains sociolinguistes, après avoir valorisé un «qualitativisme d'opposition », anti-positiviste, de revenir à des options plus quantitatives, orientées vers l'objectivation des « données » et de leur « contexte» (cf. Robillard et al. sur les notions de «terrain » et de « corpus » en SF) ${ }^{\mathrm{xvii}}$. 
La tendance semble donc à un objectivisme assumé en sociolinguistique, ce qui est contradictoire avec la conception PH du qualitatif que nous défendrons plus bas ${ }^{\text {xviii }}$.

Par ailleurs, le constructivisme est pensé par la SF dans une version que l'on pourrait qualifier de « rationaliste » ou « volontariste » (issue notamment des travaux de Piaget et Palo Alto) : ce qui est mis en avant dans la (co)« construction» du sens, c'est la rationalité des acteurs, le caractère délibéré de cette élaboration. Les courants $\mathrm{PH}$, explorés plus bas, admettent quant à eux une part d'incontrôlable dans la construction du sens, qu'il y a du sens, des connaissances qui se construisent «malgré moi et les autres», parfois de manière «cachée », sans une volonté rationnelle et explicite.

\section{Une problématisation phénoménologico-herméneutique du qualitatif}

Cette problématisation ne peut se faire qu'en ayant recours à une synthèse fortement colorée d'épistémologie, et cela pour une raison simple : comme on l'a vu plus haut, les courants qui inspirent la SF (la philosophie analytique, la pensée bourdieusienne, le pragmatisme), se sont donné des bases par le biais d'une réflexion épistémologique approfondie. La pensée phénoménologique-herméneutique $(\mathrm{PH})$ entretient une forte altérité avec ces courants (Babich, 2012) si bien que, pour la rendre discutable, il faut inévitablement exposer quelquesuns de ses linéaments, ce par quoi on commencera ici.

Ce courant a longuement et intensément débattu de la question de la qualité, si l'on considère que, étymologiquement, qualité signifie « manière d'être ».

Il a posé à nouveaux frais la question de l'«être » depuis un siècle environ, en renouvelant et en contestant l'épistémologie classique, qui, notamment est à la source de la conception à base rationaliste des phénomènes langagiers qui innerve les SHS. Cela s'est fait tant sous la plume de Heidegger que de Gadamer, pour réfléchir à la dimension langagière, linguistique, discursive de l'être comme processus individuel et social. La question de l'être est une des questions fondamentales de la tradition de pensée occidentale, que la philosophie analytique et le pragmatisme ont cru pouvoir marginaliser. D'une part, la philosophie analytique s'oriente vers la conversion des questions philosophiques, avec Frege, Russell et Wittgenstein, en termes de problèmes de langage (logique ou «ordinaire»), et en marginalisant les questions de fond telles que celle de l'être (ce courant produit la pragmatique, qui influence la SF). Le pragmatisme américain, en s'appuyant sur les travaux de Darwin et du behaviorisme, élabore un point de vue rationaliste sur la communication qui 
fait une place importante aux questions sémiotiques, et qui essaie aussi de délégitimer les questions comme celle de l'être, au fondement de la réflexion sur la qualité.

De l'autre, le courant PH constitue une autre voie, assez peu explorée par la SF, malgré son intérêt. Ces deux orientations, selon l'expression de Grondin, entretiennent une réelle « tragédie » de (mé)compréhension entre elles tant leur altérité intellectuelle est forte (Babich, 2012, p. 13).

La PH met l'accent sur le sens, et postule deux sources de sens. La première, la plus fondamentale, est l'expérience, la seconde est sémiotique (cette seconde source étant la seule véritablement prise en considération par les deux autres orientations, qui privilégient la matérialité des signifiants). Exister, vivre ne peut se faire sans attribuer implicitement du sens à ce qui n'est pas soi, parce que vivre repose sur des hypothèses sur le monde. Pour les deux autres courants, seul le sens sémiotique serait pertinent, ceci comportant des enjeux impossibles à résumer ici. Pour la $\mathrm{PH}$, le sens peut être expérientiel, implicite, indépendant de tout langage ou de toute forme sémiotique. Ce sens, par son caractère fondateur de la relation à ce qui n'est pas soi, et précisément en raison de son caractère implicite qui le rend peu objectivable, peut influencer fortement l'autre forme de sens, explicite, rationnel, lié à des signes, langages, langues, discours (désormais $L$ (Robillard, 2008). Pour la PH, tout travail du seul sens explicite, sémiotique, est toujours incomplet, parce que travaillé de l'intérieur, par le sens expérientiel implicite. La conception PH est donc contradictoire dans ses fondements avec celles des deux autres courants mais, en un sens les englobe, puisque la PH ne nie pas la pertinence du sens sémiotique qu'elle considère comme nécessaire, mais pas suffisant.

L'expérienciation de la qualité, pour la PH consisterait donc en l'expérience de la différence d'être entre soi et les autres, entre les autres entre eux, en soi-même. Pour la PH en effet, nous avons une connaissance instrumentale du monde et des autres tant que nous nous laissons emporter par l'affairement de la vie quotidienne. Dans ce rapport au monde, nous ne pouvons avoir accès qu'à la valeur instrumentale des choses et des autres humains. Il nous est difficile de sortir de ce type de relation parce qu'il a l'avantage de nous épargner le souci de notre propre être, de faire l'effort d'assumer notre histoire et de la conjuguer ainsi que notre expérience avec notre projection dans l'avenir, en rencontrant les autres non en tant qu'adjuvants de nos désirs, mais en tant qu'être eux aussi animés par le souci de soi.

Si nous parvenons à nous extraire de l'affairement, alors nous pouvons connaître le monde et les autres autrement, dans une relation qui les « dévoile » divers, d'une manière qui modifie notre propre être, puisque le processus d'être au monde ne peut se faire que dans la relation sociale avec les autres : percevoir les autres autrement ne peut que changer notre être. 
Il est intéressant, pour la sociolinguistique, que la $\mathrm{PH}$ thématise tout particulièrement le $L$ comme clé de ce processus ontologique. Pour la $\mathrm{PH}$, le $L$ est un processus ambigu, qui permet à l'expérience de chacun de se manifester aux autres «montrée-cachée». Formuler cela autrement que dans la tradition $\mathrm{PH}$ consisterait à considérer que le $L$ favorise un processus à la faveur duquel on peut comprendre les autres, leur donner sens à notre horizon, sans que le $L$ ne donne jamais les moyens d'être certain, de "prouver», que cette compréhension « émique » a eu lieu. Un être transparent aux autres serait potentiellement sous le pouvoir manipulateur des autres, à leur merci, dépourvu de toute altérité, hypothèse/objectif contradictoire avec l'éthique de la diversité posée par les SHS, et en particulier par la sociolinguistique.

Le $L$, et particulièrement les langues, jouent un rôle fondateur dans ce processus de (dé)voilement de l'être. Les langues sont à la fois une manière pour chaque être humain de s'enraciner, en le revendiquant par ses choix de langues, dans des traditions historiques qui ont donné forme à chaque langue selon des expériences héritées de sens. C'est aussi une façon de se projeter dans l'avenir, avec les autres, pour partager son expérience, raison pour laquelle Heidegger use de l'expression énigmatique selon laquelle le $L$ est la maison de l'être : le lieu qu'il habite, anime, rend vivant? Le lieu qui l'abrite? Le lieu où il peut se rencontrer ?

Si une fonction importante des SHS et de la sociolinguistique est de tenir un discours sur (et impliquant) les autres pour en faire sens, alors ce point de vue est particulièrement stimulant puisqu'il suggère de nouvelles orientations à la sociolinguistique, et partant, aux SHS, si l'on admet la place centrale du $L$ dans celles-ci (ce que reconnaissent en tout cas les trois grands courants philosophiques qui s'affrontent).

La sociolinguistique se donnerait comme fonction celle de travailler l'être, la qualité, des autres, dans des relations avec eux (donc pas de manière objectivante), en portant une attention particulière aux dimensions sociales de cet être, partiellement au moins manifestée par le $L$, l'être et le sens étant évidemment intimement liés. Selon le point de vue PH, le langage est « une recherche inassouvie du mot juste » avec les autres (Gadamer, 1996, 184).

Mais à la différence de bien d'autres conceptions fondatrices en SHS, la PH considérerait que, au-delà du $L$, ce qu'il faut entendre, c'est ce qui fait la qualité fondatrice des autres dans leur être social, que Heidegger appelle le silence sans doute parce que, à travers le $L$, il se (dé)voile, et ne se dit jamais clairement, parce que la relation sociale est sans cesse rejouée.

Sur le plan méthodologique, les propositions ne manquent pas, que l'on pense aux propositions de Jauss, de Derrida, de l'historien Kosselek, mais il serait difficile, dans l'espace imparti ici, à la fois de donner le détail de chacune de ces perspectives, et d'évoquer 
les nuances qui les opposent. Les « considérations épistémologiques et méthodologiques » de Martineau, Simard et Gauthier (2001), publiées dans cette même revue, nous semblent proches de ce que nous essayons de proposer en SF : très inspirés par l'herméneutique gadamerienne et ricordienne, ces auteurs proposent en particulier de développer des pratiques méthodologiques en SHS n'excluant pas la «pratique littéraire », le «point de vue », la «métaphore », etc. Beaucoup de points convergent avec ce que nous avons proposé comme pistes pour la prise en compte, dans les travaux des chercheurs-sociolinguistes, de ces aspects travaillés par le courant PH (Robillard, 2008 et 2009a, Debono, 2010).

\section{Conclusion}

Un regard $\mathrm{PH}$ s'intéresse, dans une activité de recherche, à sa dimension historique et à sa dimension épistémologique, les deux étant liés. Il faut alors constater que la SF, qui se conçoit qualitative, semble avoir assez largement délaissé et/ou délégué à d'autres ces deux préoccupations. Dans la perspective de la reconnaissance d'un champ, cela suscite des interrogations.

Pour que la SF soit, et soit qualitative, une réflexion épistémologique s'impose afin qu'elle puisse conjuguer et une utile centration sur des interventions et une fécondation par la fonction « laboratoire d'idées » qui distingue la recherche de l'intervention. Le point de vue $\mathrm{PH}$ intègre nombre de caractéristiques importantes pour la SF : réflexions sur la diversité, l'hétérogénéité, le changement, et surtout, le rôle ontologique des langues, langages et discours dans une société. Il s'agit d'un courant issu des travaux épistémologiques parmi les plus stimulants du $20^{\text {ème }}$ siècle, ce qui constitue déjà une forme de reconnaissance.

Il ne faut cependant pas sous-estimer que ce processus de reconnaissance, bien que pouvant se revendiquer d'une tradition ancienne et bien installée, présente une altérité forte par rapport aux courants dominants des SHS, au sein desquels l'inspiration pragmatiste et pragmatique est bien ancrée (Pudal, 2008).

En somme, la question qui se pose donc est de type «interculturel»: la sociolinguistique parviendra-t-elle à se décentrer suffisamment pour entendre la façon de poser ses questions que propose la $\mathrm{PH}$ ? Les tenants de la $\mathrm{PH}$ parviendront-ils, pour sortir la SF de son centrage autour de courants très proches les uns des autres depuis une trentaine d'années, à faire entendre l'altérité de la $\mathrm{PH}$ ? Le précédent de l'histoire du débat entre pragmatisme, philosophie analytique d'une part, et PH d'autre part (Babich, 2012) indique qu'il s'agit d'un réel défi, que la SF est sans doute capable de relever, parce que constituée en creuset 
d'influences diverses, à la fois traditionnelles et européennes (marxisme, linguistique comparée, linguistique saussurienne, dialectologie, etc.) et britanniques et nord-américaines plus récemment (pragmatique linguistique, anthropologie linguistique, ethnométhodologie, interactionnisme symbolique, etc.). Cette diversité constitutive du champ devrait donc être la garantie d'un dialogue possible avec une tradition étrangeante, dialogue qui a d'ailleurs déjà commencé (voir notamment l'échange entre Gadet, 2009 et Robillard, 2009b).

\section{Références bibliographiques}

Abric, J.-C. (1994). Pratiques sociales et représentations. Paris : PUF.

Auroux, S. (1994). La révolution technologique de la grammatisation. Introduction à l'histoire des sciences du langage. Liège : Mardaga.

Babich, B., (2012). La fin de la pensée? Philosophie analytique contre philosophie continentale. Paris : L'Harmattan.

Baggioni, D. (1986). Langue et langage dans la linguistique européenne (1876-1933), thèse de doctorat, Université de Provence, 652 p.

Blanchet, P. (2000 / 2012 (rééd.)). La linguistique de terrain. Méthode et théorie. Une approche socio-linguistique. Rennes : Presses Universitaires de Rennes.

Blanchet, P. (2002). Implications pratiques de la phonologie fonctionnelle: de la méthodologie de recherche à l'intervention didactique. La linguistique, 38, 37-52.

Blanchet, P. (2012). Intervention du réseau francophone de sociolinguistique / FRS et GIS, AIRDF, 51, 11-14.

Blanchet, P., Calvet, L-J., et Robillard (de), D. (2007). Un siècle après le cours de Saussure : la linguistique en question. Préface, Carnets d'Atelier de Sociolinguistique, 1.

Blanchet, P., Moore, D., et Asselah-Rahal, S. (2008). Perspectives pour une didactique des langues contextualisée, Paris : AUF/Éditions des Archives contemporaines.

Boghossian, P. (2009). La Peur du savoir. Sur le relativisme et le constructivisme de la connaissance. Marseille : Agone.

Boltanski, L. (2011). Le pouvoir est de plus en plus savant. Entretien avec Luc Boltanski, La vie des idées. Repéré à : http://www.laviedesidees.fr/Le-pouvoir-est-de-plus-enplus.html.

Bourdieu, P. (1997). Méditations pascaliennes. Paris : Seuil.

Bourdieu, P. (2001). Langage et pouvoir symbolique. Paris : Seuil. 
Bourdieu, P. et Passeron, J.-C. (1970). La Reproduction : éléments d'une théorie du système d'enseignement. Paris : Les Éditions de Minuit.

Boutet, J. (1994). Construire le sens. Bern : Peter Lang.

Boutet, J. (2000). Courants en sociolinguistique française, Sociolinguistica. 14, 214-224.

Boutet, J. (2006). Article « Sociolinguistique» in Mesure / Savidan, éds., Le dictionnaire des sciences humaines. Paris : PUF.

Boutet, J. (2010). Histoire de la sociolinguistique en France : quelques jalons et filiations. Dans Gasquet-Cyrus, M., Giacomi, A., Touchard, Y. et Véronique, D. (Éds). Pour la (socio)linguistique (pp. 59-76). Paris : L'Harmattan.

Boutet, J. (2010). Du travail entre disciplines. Dans Dunezat, X., Heinen, J., Hirita, H. et Pfefferkorn, R., Travail et rapports sociaux de sexe. Rencontres autour de Danièle Kergoat. Paris : L'Harmattan.

Boutet, J., et Tabouret-Keller, A. (Éds). 2009. Marcel Cohen : aux origines de la sociolinguistique. Langage et société, 128. Repéré à : http://www.cairn.info/revuelangage-et-societe-2009-2-page-5.htmr

Boyer, H. (1990). Matériaux pour une approche des représentations sociolinguistique. Eléments de définition et parcours documentaire en diglossie. Langue française, 85, 102-124.

Boyer, H. (2001). Introduction à la sociolinguistique. Paris : Dunod.

Boyer, H. (Éd). (1996). Sociolinguistique : territoire et objets. Lausanne-Paris : Delachaux et Niestlé.

Boyer, H. (Éd). (2010). Pour une épistémologie de la sociolinguistique. Actes du colloque international de Montpellier. Limoges : Lambert-Lucas.

Boyer, H. et Prieur, J.-M. (1996). La variation sociolinguistique. Dans Boyer, H. (éd.). pp. 3568.

Bulot, T., Bauvois, C. et Blanchet, Ph. (Éds). (2001). Sociolinguistique Urbaine. Variations linguistiques : images urbaines et sociales. Cahiers de Sociolinguistique, $\mathrm{n}^{\circ} 6$.

Calvet, L.-J. (1997). Méthodes. Dans Moreau, M.-L. (Éd), Sociolinguistique. Concepts de base (pp. 211-212). Bruxelles : Mardaga.

Calvet, L.-J. (1999). Aux origines de la sociolinguistique, la conférence de sociolinguistique de l'UCLA (1964). Langage et Société. 88, 25-57.

Calvet, L.-J. (2000). Du passé au futur : quel avenir pour la (socio)linguistique française?. Sociolinguistica, 14, 78-82. 
Calvet, L.-J. (2007). Pour une linguistique du désordre et de la complexité. Carnets d'Atelier de Sociolinguistique, 1. Repéré à : http://www.upicardie.fr/LESCLaP.

Calvet, L-J. (2011 [1993]). La sociolinguistique. Paris : PUF.

Certeau (de), M., Julia, D., et Revel, J. (1975). Une politique de la langue. La Révolution française et les patois. Paris : Gallimard.

Debono, M. (2010). Construire une didactique interculturelle du français juridique: approche sociolinguistique, historique et épistémologique. Thèse. Université de Tours.

Derrida, J. (1967). L'Ecriture et la différence. Paris : Seuil.

Duschesne, S. (à paraître). Développement de l'analyse secondaire et des méthodes d'analyse qualitative : une chance à saisir ?. Dans Brugidou, M., Dargentas, M., Le-Roux, D., et Salomon, A.C. (Éds). Analyse secondaire en recherche qualitative: enjeux pour les sciences humaines et sociales. Paris : Éditions Lavoisier.

Gadamer, H.-G. (1996). Vérité et méthode. Paris : Seuil.

Gadet, F. (2005). 1977 : sur un moment-clé de l'émergence de la sociolinguistique en France. Cahiers de l'ILSL, 20, 127-138. Repéré à : http://www2.unil.ch/slav/ling/recherche/biblio/05MarrCB/Gadet.pdf

Gadet, F. (2009). Lecture critique de l'ouvrage de Didier de Robillard Perspectives alterlinguistiques. Langage et société, 128, 143-154.

Garrau, M., Le Goff, A. (Éds). (2009). La reconnaissance : perspectives critiques, Nanterre : Université de Paris X.

Goï, C. et Pierozak, I. (2010). Le « terrain » : en long, en large et en travers. Dans Pierozak, I., (Éd.), Du «terrain » à la relation: expériences de l'internet et questionnements méthodologiques. Cahiers de linguistique, 36(2), 17-28.

Heidegger, M. (2008). La logique comme question en quête de la pleine essence du langage. Paris : Gallimard.

Jauss, H.-R (1978). Pour une esthétique de la réception. Paris : Gallimard.

Jewsiewicki, B. et Létourneau, J. (1996). L'histoire en partage : usages et mises en discours du passé. Paris : L’Harmattan.

Klinkenberg, J.-M. (1997). Classe sociale des élèves. Dans Moreau, M.-L. (Éd), Sociolinguistique. Concepts de base (pp. 81-85). Sprimont : Mardaga.

Koerner, K. (1976). Toward a History of Modern Sociolinguistics. American Speech, 6(1), 57-70. 
Kosselek, R. (1997). L'expérience de l'histoire. Paris : Gallimard / Seuil.

Laks, B. (1984). Le champ de la sociolinguistique française de 1968 à 1983. Production et fonctionnement. Langue française, 63, 103-128.

Pelletier, M. L., et Demers, M. (1994). Revue des sciences de l'éducation, 4(20), 757-771. Repéré à : http://www.erudit.org/revue/RSE/1994/v20/n4/031766ar.pdf

Marcellesi, J.-B. (1980). De la crise de la linguistique à la linguistique de la crise : la sociolinguistique. La Pensée, 209, 5-21.

Marcellesi, J.-B. et Gardin, B. (1974). Introduction à la sociolinguistique. Paris : Larousse.

Marcellesi, J.-B., Bulot, T., et Blanchet, P. (2003). Parcours d'un sociolinguiste : de la langue corse au discours politique. Dans Marcellesi, J.-B., Bulot, T., et Blanchet, P., Sociolinguistique. Epistémologie, langues régionales, polynomie. Paris : 1'Harmattan.

Martineau, S., Simard, D. et Gauthier, C. (2001). Recherches théoriques et spéculatives : Considérations méthodologiques et épistémologiques. Recherches Qualitatives, 22, 332.

Milner, J.-C. (1995). Introduction à une science du langage, Paris : Seuil.

Morin, E. (1986). La méthode. 3. La Connaissance de la Connaissance. Paris : Seuil.

Mucchielli, A. (Éd.). (2004). Dictionnaire des méthodes qualitatives en sciences humaines, Paris : Armand Colin.

Oliveau-Statius, S. (2006). Pourquoi « la langue » n’existe-t-elle plus ?. Le Télémaque, 30, 27-42.

Pierozak, I. (2011). Les corpus électroniques en sciences du langage : un eldorado ? Le discours et la langue, 2.1, 15-32.

Pierozak, I., (Éd.), (2010). Du «terrain» à la relation: expériences de l'internet et questionnements méthodologiques. Cahiers de linguistique, 36(2).

Pudal, R. (2008). La sociologie français est-elle pragmatist compatible ? Tracés, №15, 25-45.

Rastier, F. (2001). Arts et sciences du texte. Paris : PUF.

Rastier, F. (2009). Saussure et les textes. De la philologie des textes saussuriens à la théorie saussurienne des textes. Texto !, 14(3), 1-26. Repéré à : http://www.revuetexto.net/index.php?id=2327.

Razafimandimbimanana, E. (2005). Français, franglais, québé-quoi? Les jeunes Québécois et la langue française : enquête sociolinguistique. Paris : l'Harmattan.

Ricœur, P. (1990). Soi-même comme un autre. Paris, Seuil.

Robillard (de), D. (2007). La linguistique autrement : altérité, expérienciation, réflexivité, 
constructivisme, multiversalité : en attendant que le Titanic ne coule pas. Carnets d'Atelier de Sociolinguistique, 1. Repéré à : http://www.upicardie.fr/LESCLaP/IMG/pdf/robillard_CAS_no1.pdf.

Robillard (de), D. (2008). Perspectives alterlinguistiques, vol. 1 - Démons, vol. 2 Ornithorynques. Paris : L'Harmattan.

Robillard (de), D. (Éd.). (2009a). Réflexivité, herméneutique: vers un paradigme de recherche? Cahiers de sociolinguistique 14. Rennes : Presses Universitaires de Rennes.

Robillard (de), D. (2009b). Ce que 'comprendre' pourrait bien vouloir dire. Langage et société, $\mathrm{n}^{\circ} 130,125-136$.

Robillard (de), D. (2010). Fallait-il inventer la sociolinguistique moderne ? Enjeux autour de l'histoire et de la sociolinguistique. Dans Gasquet-Cyrus, M., Giacomi, A., Touchard, Y. et Véronique, D. (Éds). Pour la (socio)linguistique (pp. 77-92). Paris : L’Harmattan.

Robillard (de), D., Debono, M., Razafimandimbimanana, E., et Tening, M.-L. (à paraître). Le sociolinguiste est-il (sur) son terrain? Problématisations d'une métaphore fondatrice. Colloque du Réseau Francophone de Sociolinguistique, Dynamiques plurilingues : des observations de terrains aux transpositions politiques, éducatives et didactiques, Alger, 31 mai, 1 er et 2 juin 2011.

Saussure (de), F. (2010 [1916]). Cours de linguistique générale. Paris : Payot.

Savatovsky, D. (2011). Les pratiques de qualification au sein de la 7e section du CNU (20052011). Repéré à : http://assoc-asl.net.

Sokal, A., et Bricmont, J. (1997). Impostures intellectuelles. Paris : Odile Jacob.

Taylor, C. (1997). La liberté des modernes (chap. "L'interprétation et les sciences de l'homme », pp.137-194). Paris : PUF, 1997.

Trimaille, C. (2003). Approche sociolinguistique de la socialisation langagière d'adolescents. Thèse de doctorat, Université Stendhal - Grenoble III.

Van den Berg, H. (2005). Reanalyzing qualitative interviews from different angles: the risk of decontextualization and other problems of sharing qualitative data. Forum: Qualitative Social Research, 6(1). Repéré à : http://www.qualitative-research.net/

Von Glasersfeld, E. (1994). Pourquoi le constructivisme doit-il être radical ?. Revue des sciences de l'éducation, 20(1), 21-27.

Wittgenstein, L. (2005 [1953]). Recherches philosophiques. Gallimard. 
${ }^{\mathrm{i}}$ Nous distinguerons 1'« Histoire », à visée linéaire, universelle et conventionnelle, d'une « histoire » plurielle, interprétative, problématisée, et donc débattue.

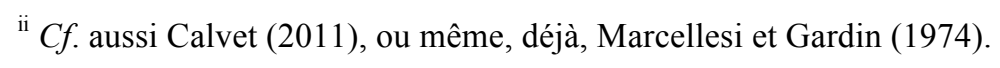

iii Quelles que soient les listes établies, sont lisibles des enjeux de (non-)reconnaissance (ex. : l'oral est mis en avant à la différence des représentations (Laks, 1984)), et donc de (non-)reconnaissance disciplinaire (ex. sociologie du langage, analyse du discours, etc).

${ }^{\text {iv }}$ Il est frappant par contre de trouver, en lien avec des langues minoritaires, l'affirmation de sociolinguistique par exemple « catalane», etc. Cela pourrait donc se lire (ailleurs qu'ici) au regard de la politique linguistique française et de sa tendance à universaliser le cas français.

${ }^{v}$ Or un champ a-t-il à présenter un visage nécessairement homogène ? Ce serait comme sciemment mettre à l'écart toute forme de dynamisme, de débats et d'altérité.

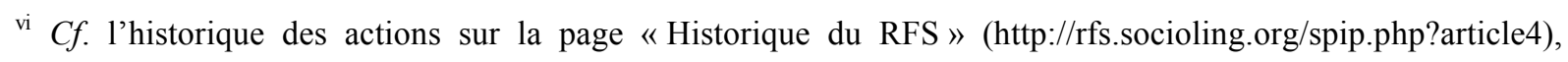
consultée en mars 2012.

vii «[La] fibre «critique» [a] un peu tendance à se faire discrète dans la sociolinguistique française de ces dernières années, du moins au plan épistémologique, souvent oublié derrière l'urgence impérieuse de la demande sociale. » (nous soulignons) (compte-rendu de l'ouvrage de Robillard (de), 2008 : Gadet, 2009, p. 152). viii « L'expérience (générique) oppose l'expérimentation (manipulation, hiérarchie) à l'expérienciation (participation, parité).» (Robillard (de), 2007, p. 32).

${ }^{\text {ix }}$ Si les méthodologies qualitatives de la SF sont implicites et peu problématisées, cela peut en effet s'expliquer par une constitution historique de la discipline dans un double mouvement corrélé d'opposition à sa première discipline tutélaire (la linguistique) et d'emprunt à d'autres disciplines (sociologie et anthropologie en particulier) : comme le remarque Calvet, la sociolinguistique «n'a pas [...] inventé grand-chose » du point de vue des méthodologies (in Moreau, 1997, p. 211). Il ne s'agit pas ici de porter un quelconque jugement sur cette fondation interdisciplinaire des méthodologies en SF, mais de remarquer qu'elle explique en partie le fait qu'une réflexion propre sur une conception sociolinguistique du qualitatif n'ait que très peu été menée.

${ }^{\mathrm{x}}$ L'ouvrage édité par H. Boyer (2010) à la suite d'un colloque portant spécifiquement sur l'épistémologie de la sociolinguistique ne fait, dans l'ensemble, que très peu référence aux problèmes épistémologiques généraux, pour se consacrer à la discussion de notions sociolinguistiques ponctuelles.

${ }^{x i}$ Parmi les dix caractéristiques essentielles des méthodes qualitatives, les deux premières sont : 1) l'induction à partir de données recueillies (vs l'analyse des données pour vérifier des hypothèses posées a priori) ; 2) l'étude du contexte écologique dans lequel évoluent les sujets ou groupes étudiés (Taylor et Bodgan 1984, cités par Mucchielli, 2002, p. 58).

${ }^{x i i}$ La question de la valeur de l'empirisme est largement discutée depuis plusieurs siècles. Rappelons brièvement la mise en place des traditions idéalistes (Platon, Descartes, Kant) et empiristes, la plus connue étant celle de l'empirisme anglais (Bacon, Hobbes, Locke, Hume), débat auquel se rajoutent plus tardivement par exemple 
Duhem et Quine qui, chacun à sa façon, argumentent en faveur de l'impossibilité de dissocier théorie et données fournies par l'expérience.

xiii "context is used in everyday discourse as a rhetorical device to undermine undesired interpretations and to impose desired interpretations of someone's utterances" (Van den Berg, 2005).

${ }^{\text {xiv }}$ La question de la limitation de l'interprétation par la contextualisation se pose avec une acuité particulière quand il s'agit de déterminer l'éventuelle possibilité du partage des données et de l'analyse secondaire de corpus établis selon une démarche qualitative (Duchesne, à paraître).

${ }^{\mathrm{xv}}$ «[...] la dynamique d'une situation linguistique donnée ne peut être appréciée qu'au prix d'un repérage attentif des pratiques et des représentations sociolinguistiques » (Boyer, $2001: 45$ ). « Il nous faut donc accepter de considérer la 'langue' comme un ensemble de pratiques, souvent désordonnées, et comme un ensemble de représentations, celles des locuteurs, bien sûr, mais aussi celles des linguistes, et chercher à comprendre comment fonctionne ce désordre » (Calvet, 2007, p. 47).

${ }^{\text {xvi }}$ Et la possibilité d'une tierce voie entre objectivisme et subjectivisme est exclue. Voir l'intéressante réflexion en science de l'éducation de Martineau et. al. (2001: 10-12).

xvii Tentation que l'« eldorado» des corpus numériques (Pierozak, 2011) ne fait qu'accentuer : la masse des données à traiter amène des discours sur la nécessaire articulation des approches qualitatives et quantitatives qui virent bien souvent à l'avantage de ces dernières.

xviii « la perspective herméneutique permet de proposer des principes communs pour récuser l'objectivisme, la réduction de la description scientifique à l'explication causale et l'ontologie spontanée du représentationnalisme» (Rastier, $2001: 100)$. 